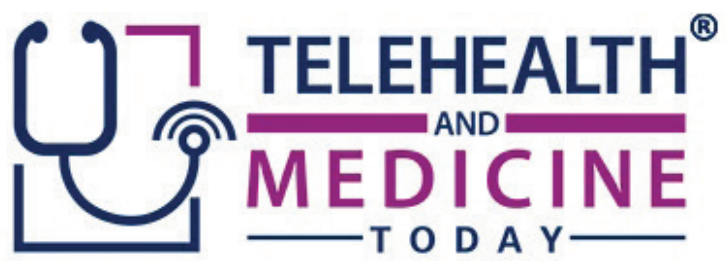

\title{
Assessing the remote management of Family Medicine education during the SARS-CoV-2 Pandemic
}

\author{
Pedro J. Tarraga Lopez, PhD MD ${ }^{1}$, Lourdes Navarro Sanchez, $\mathrm{PhD}^{2}$, \\ Ibrahim M. Sadek ${ }^{3}$
}

Affiliations: ${ }^{1,2}$ Associate Professor of Medicine University of Castilla la Mancha; ${ }^{3}$ Health Care Medical Albacete.

Corresponding author: Pedro J. Tárraga Lopez; Email: pjtarraga@sescam.jccm.es

Keywords: Telematics; Online Classes; Audiovisual Media; Medical Education; Remote Education

Section: Original Clinical Research

Introduction: The SARS-CoV-2 (COVID-19)

coronavirus pandemic has changed lifestyles, work styles and, of course, training and teaching.

Objective: To evaluate the telematic training of the subject of family medicine at the University of Castilla la Mancha.

Methods: it was proposed to implement a methodology of telematic classes, allowing the student to self-manage their time and receive the contents at distance. The acceptance of the new methodology was subsequently evaluated through satisfaction surveys and the effect of the methodology on the students' grades was evaluated comparatively.

Results: Favorable results were obtained with a global average for the course of 4.95 out of 5 for the year 2020 and 4.85 and 4.87 for the years 2018 and 2019. The specks obtained above all in the theory exam are higher than that of these years.

Conclusion: It is concluded that the telematic approach is a learning methodology valued positively by the students with a favorable impact on their academic results.

\section{INTRODUCTION}

The COVID-19 pandemic is categorized as the greatest crisis that the human species has faced in the last two centuries, comparable only with situations of the caliber of World Wars. It is a multifactorial problem that affects the deepest social, educational, economic and, of course, public health dimensions of our lives.

In the educational system, a forced and vertiginous challenge was imposed on us to 
quickly adapt our teaching to an online format, including those educational levels that we would never have thought of teaching completely at distance, such as kindergarten, primary, high school and even university has had to reinvent itself to persist in this pandemic.

Regarding the transformation of the higher education programs and especially the training of health professionals, we allow ourselves to pose some reflections on the implementation of the online learning modality, which are concluded from the lectures of the faculties of Medicine of our university shortly after the crisis, and which has been shared by the world medical community.

Conceptually, telematic education is defined as the use of the Internet for educational purposes. It is a teaching-learning modality that aspires to be flexible, attractive and centered on the student, fostering collaboration, communication and interaction between the members of the learning community (students, tutors and teachers) with the content. Therefore, it is more than a repository of resources that the teacher has to be reviewed by students during a certain period of time. ${ }^{1}$ Digital resources are organized in virtual educational environments and should be considered a means for learning and not an end in itself: readings, presentations, videoconferences, blogs, wikis, social networks, forums, podcasts, portfolios, games, simulations, among others.

Likewise, there is a continuum between a completely face-to-face modality until online courses, passing through an intermediate strategy known as "blended" or hybrid that combines both modalities, ensuring that much of the theoretical content is approached remotely and face-to-face meetings are reserved for those learning experiences that require face-to-face interaction, such as clinical activities, field visits, clinical reasoning seminars, laboratories, among others.
The online modality offers multiple advantages for the training of health students, such as promoting self-directed learning, through flexible learning environments with permanent availability, as well as involving students in a collaborative learning community ${ }^{2}$. Technology is revolutionizing medicine; however, the way of training health professionals has not changed with the times. It is true that clinical medicine is learned in the clinical environment and the encounter with the patient is very valuable as an educational experience, but we have been ineffective as educators in time management and human resources. Online resources can be used to prepare efficient clinical experiences with high educational impact ${ }^{3}$.

In this context of a pandemic, where the design and implementation of virtual teaching has had to be done with haste and not without problems, it has been essential to learn from other experiences. In this sense, a group of medical educators from the United Arab Emirates makes some recommendations for their adoption in an emergency context, in order to successfully cope with the change of the learning modality in these times, which are: a) establish a sense of urgency, which is crucial for managing the change, $b$ ) establish a working group within the faculty management team to lead the transition from face-to-face to online learning modality, c) carry out an analysis of teachers' and students' needs (digital skills, resources and equipment, among others), d) establish and communicate a work plan to teachers and students, e) train teachers in the roles of virtual teacher: pedagogical, administrator, social and technical, $\mathrm{f}$ ) agree on teaching content, deciding what can be taught now, what should be added, omitted or postponed, g) establish content delivery strategies (synchronous or asynchronous), h) cope with the stress of the students, by both, learning modality and by the health emergency 
situation and its personal and / or family repercussions, i) promote the motivation and commitment of the students, j) plan the evaluations and exams, $\mathrm{k}$ ) anticipate problems and solution strategies (connectivity problems, instability of communication platforms, difficulties that teachers in particular might find, among others) and 1) monitor and evaluate the implementation of the educational approach.

In this scenario of change, it is the clinical environment that is proportionally most affected, given mainly by its way of learning through interaction with patients in the real context.

Among the reasons put forward to explain the deterioration of clinical teaching is the shortage of teaching time, stress and anguish of clinicians who participate directly in the care of patients in the pandemic and the definitive suspension of the undergraduate clinical practices in the hospitals of our university. Faced with these difficulties, the following questions and ideas arise to successfully compensate for clinical training; 1) promote a feeling of new normality to students, motivated by a tutor with leadership skills, 2) implement clinical case resolution in virtual sessions, 3) make the most of digital health tools, beyond just telemedicine, 4) train the student in the management of symptoms, signs and complementary tests through the interrelation through the internet and 5) respect for bioethical standards required by the context of digital health ${ }^{3}$.

The implementation of virtual teaching implies multiple challenges, both for teachers and students. On the one hand, teachers trained in planning, design, implementation and evaluation in virtual educational environments are required. This implies breaking the temptation to carry out the same classroom on a virtual platform.

Modifying the course plan creatively will allow the use of the best of digital tools to promote deep, collaborative, and meaningful learning. Given the above, it is imperative to incorporate these topics in the training programs of future educators of the health professions: instructional design, creation of digital resources, evaluation in virtual environments, effective feedback, among others. On the other side, students studying subjects with an important virtual component require developing the capacity for self-regulation and effective management of working time, in order to properly manage their own learning process, and academic integrity, especially in the evaluation processes and generation of products that serve as evidence of learning.

The COVID-19 pandemic has driven a digital transformation of education at all levels, for which we were probably not prepared, but we sensed that it must have occurred a long time ago.

Within the barriers of implementation of this educational modality, the problem of inequity that has dragged our educational system for several years is a factor that makes it difficult, situations such as the high percentage of households without an internet connection and problems with the availability of computers for students, especially in remote and rural places, are situations that must be corrected.

But in spite of everything, it is true that it is not possible to conceive a curriculum completely online, especially in undergraduate training, however, we can incorporate the good practices learned during this period, to implement them in our teaching methods the day the pandemic is overcome. Thus, it will have been a virus that, among other things, has produced the education revolution, achieving a teaching according to the times, focused on the student and supported 
by digital resources, without forgetting that the final purpose of all our work is the training of doctors.

\section{METHOD}

Medicine studies in Europe include undergraduate studies that are taught at the university and in recent years in health centers and specialty studies (Intern Resident Physician) that are developed in hospitals and other health centers during $4-5$ years.

Implementation of the course:

Firstly, the Family Medicine course is taught in the second semester of the fifth year with a structure of 5 modules on the topics:

I) Organization and Structure of Primary Health Care in Spain and other countries.

II) Addressing Family problems and community care.

III) Approach to chronic diseases patient.

IV) Approach to the terminal patient and home consultations.

V) Addressing immigration and gender violence.

And 5 seminars on other topics:

I) Cardiovascular Risk Management.

II) Approach to Mental Health in Primary Care.

III) Clinical Interview and Doctor-Patient relationship.

IV) Approach to Pain in Primary Care.

V) Health Prevention and Promotion Activities

An important part of the program are the practices that are carried out in the Health Centers with real patients for a week.

The start of the pandemic coincided after the delivery of blocks I and II and seminars I and II.
The restructuring of the course was divided into a design phase, in which the oriented course program was created in an online format. To do this, the traditional course program (objectives and contents) was reviewed, and the classes that would be adapted to the online format were defined, and those that would be held in person; which would have an online course.

Next, for the classes in online format, the pedagogical resources were defined (virtual space Moodle and Microsoft Teams of the University of Castilla la Mancha) allowing to deliver the contents and achieve the learning objectives. Then, the program for online classes was written. Proceeding to teach each of the classes and select audiovisual resources. For the recording and editing phase of audiovisual media, the team designated by the Dean's Office and the staff of the Medical Education Unit were supported by the University's tech support unit.

A total of 12 classes were given online for the topics of modules III and IV and seminars III and IV.

The Microsoft Teams platform was used for online practices that replaced face-to-face practices to carry out these practices, with a standard format for each presentation that included the presentation of clinical cases of:

I) Clinical interview

II) Patients with chronic diseases.

III) Patients with cardiovascular diseases.

IV) Patient with psychosocial diseases with family and / or community involvement.

V) Patients with various types of pain.

In all cases, the students interacted with the teacher responsible for the practice, who made the presentation of the content and even some video taken from various sources (TV series, 
movies, popular culture) to reinforce, exemplify or propose an analysis.

The classes, tried in the most effective way to emulate a real lesson class (although, in this case, asynchronous) consisted of a standard presentation and a small video at the bottom of the screen, where the teacher appears dictating the instructional material that unfolds.

These classes were on the Teams platform of the course according to a previously designed calendar and could be reviewed by the student.

Course evaluation: To evaluate the delivered syllabi, students were subjected to online questionnaires of 60 multiple choice questions, development that included critical analysis, definition of concepts, discussion of cases, etc., which were uploaded to the web platform of the course the time of day intended for said evaluation, giving a period of 1 hour for its completion.

Subsequently, they were given a satisfaction survey of 5 questions about the subject. The surveys were anonymous and with prior consent.

\section{Ethical aspects}

Given that anonymity was guaranteed in the questionnaire to be completed by the participants and therefore no personal data would be collected, it was not necessary to sign the written informed consent. Before starting the questionnaire, the objective of the research was explained to the participants and their permission was requested to publish the study data.

The study has been carried out in accordance with the Declaration of Helsinki, and the collection of the data has been anonymous following the guidelines of the Spanish Organic Law on Protection of Personal Data (LOPD) 15/1999.

\section{Analysis of data}

The statistical analysis has been carried out with the statistical program SPSS version 24, 103 University of Castilla la Mancha license. From the computer platform used to collect the necessary. 112 data for the present study, the variables were ordered, coded and refined to be able to be analyzed.

Qualitative variables are presented as absolute and relative frequencies. The quantitative ones as mean and standard deviation (SD). The relationship between the qualitative variables was made using the chi-square test or Fisher's exact test in case more than if in a $2 \times 2$ table there were more than $20 \%$ had an expected frequency lower than 5. The comparison between the means of the quantitative variables, when they had two categories, it was performed with Student's $t$ test for independent groups after checking the homogeneity of variances with Levene's test or Mann-Whitney's U if the behavior of the variable was not normal. When the quantitative variables were related, we used t- Student's paired test, or Wilcoxon test if the behavior of the variable was not normal. When the groups had more than two categories, the analysis of variance (ANOVA) or the non-parametric Kruskal-Wallis test was used if the normality conditions were not met. In case of obtaining significant results, to observe between which groups the significant differences are found, the Bonferroni a posteriori test would be applied.

\section{EVALUATION TO COMPLETE THE 5TH COURSE STUDENT (2019-2020)}

Indicate the degree of satisfaction (1: Not at all satisfied to 5: Very satisfied), in each of the aspects indicated below:

Planning of teaching activities 12345

Monitoring and individualized support for your training 12345 
Adequacy of the teaching program 12345

Satisfaction with the tutor's work 12345

Your global experience at the health center 12345

Interest for future clinical practice 12345

What aspects would you highlight positively?

What aspects do you think need improvement?

Impact of the intervention: To measure the impact of restructuring the online teaching that was given in the redesigned family medicine course, a comparative analysis was made with the previous course that was implemented with traditional teaching.

\section{RESULTS}

117 students are enrolled in the Family Medicine course, although 2 have studied at other universities (SENECA ...), of the 115 participants only 34 are men.

Attendance to online classes, being more comfortable and accessible due to being at home, has been in the majority between $95-100 \%$ of the total higher than other previous courses, which is between $80-90 \%$. Attendance at practical seminars is similar. Table 1.

Regarding the qualifications obtained, it can be seen in Table 2 that there has been an increase in the grades in the theoretical exam over the two previous courses, being the practical one more similar.

Table 1. Attendance to Classes and Seminars.

\begin{tabular}{|l|c|c|}
\multicolumn{1}{|l}{ Course } & \multicolumn{1}{c}{$\begin{array}{c}\text { Attendance } \\
\text { to class }\end{array}$} & $\begin{array}{c}\text { Attendance } \\
\text { to Practices }\end{array}$ \\
\hline $2019 / 2020$ & $96.50 \%$ & $98 \%$ \\
\hline $2018 / 2019$ & $82 \%$ & $100 \%$ \\
\hline $2017 / 2018$ & $86 \%$ & $100 \%$ \\
\hline & $\boldsymbol{P}<\mathbf{0 . 0 0 2}$ & $\boldsymbol{p}<0.23$ \\
\hline
\end{tabular}

Regarding the Satisfaction survey, the score was 4.95 out of 5 and the valuations were equal to or higher than previous years, which were 4.85 and 4.87, respectively, so for questions Table 3:

\section{DISCUSSION}

The COVID-19 pandemic is the greatest crisis that the human species has faced and has forced us to change habits and lifestyles, also due to the way of working and teaching.

The completion of the family medicine course in telematic format has had promising results for medical education. The general evaluation of the students of the course has shown significant progress, being reflected in the responses to the satisfaction survey, validated by statistical methods. Among the results of the questionnaire, it should be noted that the two statements with the greatest clinical application refer to the use of extra audiovisual elements that "reinforce the

Table 2. Theory and practical notes

\begin{tabular}{|l|c|c|}
\multicolumn{1}{l}{ Course } & $\begin{array}{c}\text { Theoretical } \\
\text { Note }\end{array}$ & $\begin{array}{c}\text { Practical } \\
\text { Notes }\end{array}$ \\
\hline $2019 / 2020$ & 8.20 & 8.55 \\
\hline $2018 / 2019$ & 7.35 & 8.72 \\
\hline $2017 / 2018$ & 7.44 & 8.25 \\
\hline & $\boldsymbol{P}<\mathbf{0 . 0 3}$ & $\boldsymbol{p}<0.34$ \\
\hline
\end{tabular}

Table 3. Course Evaluation

\begin{tabular}{|l|}
\hline Teaching activities planning: Average 4.85 \\
\hline Monitoring and individualized support for \\
your training: Average 4.91 \\
\hline Adequacy of the teaching program: Average 5 \\
\hline Satisfaction with the work of the tutor \\
Average 4.92 \\
\hline Your global experience at the health center: \\
Average 5 \\
\hline Interest for future clinical practice: Average 5 \\
\hline
\end{tabular}


content" and the opportunity to "self-manage learning times". In addition, a better overall grade for the course is observed in the telematic 2019/2020 year versus face-to-face 2018/2019 and 2017/2018; Considering that the contents were maintained, the blended or the hybrid modality can be assessed, which includes online format classes and face-to-face meetings as a reinforcement and deepening, this academic improvement. It is interesting the value that the student places on the inclusion of material that is striking and familiar to him in the learning process. Similarly, the great value that the student assigns to time flexibility and the ability to distribute their free time is highlighted, which seems to be key both in the proposal of the new curriculum mesh and in the adaptation process of a student in transition to university life. This gives way to a new configuration of the student teacher interaction, where it is mainly constituted by a space for solving doubts and not merely reading, allowing the student to spend less time in the classroom without this jeopardizing the delivery of minimal content. Although it could be considered a valid argument, this, finally, contributes to further sustain the need to optimize the way in which the less valued content by the students are delivered, in a way that is efficient both for them and for the institution itself, and common learning objectives are achieved. In any case, contrary to this argument, one could use answers of an equally subjective nature, but based on our study, having maintained the quantity and quality of content and without substantial differences in the ratings - a truly essential point in the appreciation of the medical student-, the improvement in perception can be attributed quite surely to the environment in which the course is developed.

It can be mentioned that during the online evaluations there are isolated cases of irregular behavior on the part of the students regarding cheating and intercommunications with other classmates and evident content; It is open to debate about the maturity, capacity and integrity of a medical student to carry out this type of formal activity, which in turn may be specific cases since, evidently, it was not the keynote of the course. It seems quite evident that the integration of multimedia material: videos, texts, images, audio, and the ubiquity of the educational process are characteristics that have only recently been taken advantage of in pursuit of the development of more binding and holistic educational models. Which presupposes opportunities, but also challenges. Relatively recent data from the European Union show that between 50 to $80 \%$ of students in these countries have never used digital texts, exercise software, podcasts, simulation games, this added to the reluctance of teachers at least primary and secondary to be able to efficiently use these elements ${ }^{6}$; But in a rapidly growing student population, it is essential to adapt traditional learning methods or at least offer a face-to-face mixed with online format, in the usual way. This challenge is also a challenge for education scientists and experts, since there is relatively little knowledge on how to use social media and technological elements to develop a more meaningful, effective and attractive education, versus the vast knowledge regarding the potential of technology as a channel of entertainment and recreation.

The difficulty to students in accepting these disciplines is eventually justified by the absence of guidelines in most medical schools. Therefore, an effort is required to increase the quantity and quality of social skills teaching in physician education and training. ${ }^{7}$ It is also necessary to change the medical culture, taking into account the various facets and normative character of the paradigms on health, disease and health practice, or at least gain the ability to question these paradigms and develop critical thinking. ${ }^{8}$ 
Funding Statement: No financing

Conflict of Interest: No conflict of interest of any author

Contributors Contributions: The authors have contributed to the development of the article and want to thank the rest of the teachers for their collaboration.

\section{REFERENCES}

1. Ellaway R, Masters K. AMEE Guide 32: E-Learning in medical education. Part 1: Learning, teaching and assessment. Medical Teacher 2008;30:455-73

2. Lewis KO, Cidon MJ, Seto TL, et al. Leveraging e-learning in medical education. Current Problems in Pediatric and Adolescent Health Care 44: 150-63.

3. Rose S. Medical Student Education in the Time of COVID-19. JAMA 2020;323: 2131-32.

4. Taha MH, Abdalla ME, Wadi M, et al. Curriculum delivery in Medical Education during an emergency: A guide based on the responses to the COVID-19 pandemic. MedEd Publish 2020;69.

5. Wilkinson D. The future of medical education: all about being connected. The Ochsner Journal 2012;12:300-1.

6. Liyoshi T, Kumar MSV Opening Up Education . The MIT Press 2016:504.

7. Isaac M, Rief W. Role of behavioural and social sciences in medical education. Curr Opin Psychiatryc 2009;22:184-7.

8. Wong G. The curse of paradigms? Med Educ. 2012;47:333-41.

Copyright Ownership: This is an open access article distributed in accordance with the Creative Commons Attribution Non Commercial (CC BY-NC 4.0) license, which permits others to distribute, adapt, enhance this work non-commercially, and license their derivative works on different terms, provided the original work is properly cited and the use is noncommercial. See: http://creativecommons. org/licenses/by-nc/4.0. 